\title{
PRÁCTICA DELIBERADA DE PSICOTERAPIA EN EL PROGRAMA DE PSICÓLOGO INTERNO RESIDENTE ESPAÑOL
}

\section{DELIBERATE PRACTICE OF PSYCHOTHERAPY IN THE SPANISH RESIDENT INTERN PSYCHOLOGIST PROGRAM}

\author{
Pau Sánchez Rotger \\ Psicólogo clínico, Hospital Universitario La Paz. Madrid, España \\ ORCID: https://orcid.org/0000-0003-2730-8454 \\ Ignacio Serván García \\ Psicólogo clínico, director del Centro Especializado en Psicoterapia y Apego (CEPA). \\ Madrid, España \\ ORCID: https://orcid.org/0000-0002-9837-5630
}

Cómo referenciar este artículo/How to reference this article:

Sánchez Rotger, P. y Serván García, I. (2020). Práctica Deliberada de Psicoterapia en el Programa de Psicólogo Interno Residente Español. Revista de Psicoterapia, 31(116), 247-261.

https://doi.org/10.33898/rdp.v31i116.346

\section{Resumen}

A pesar de la eficacia de la psicoterapia, existe evidencia sobre sus limitaciones y posibilidades de mejora. Atendiendo al factor del terapeuta, parece que la experiencia clínica, la supervisión tradicional o la formación en tratamientos empíricamente validados no son en sí mismos y por separado una garantía de mayor eficacia. Se han diseñado instrumentos de monitorización rutinaria del resultado de la psicoterapia que pueden incrementar su eficacia. El uso de estos métodos es relevante dentro de un contexto de práctica deliberada. Este tipo de práctica se caracteriza por el establecimiento de objetivos y el entrenamiento en habilidades que exceden el nivel actual del practicante. Dentro del campo psicoterapéutico, la práctica deliberada puede contribuir a un aumento de la eficacia del terapeuta a lo largo del tiempo. Este artículo realiza una revisión de estos conceptos y propone un modelo de práctica deliberada aplicable al contexto del programa PIR español, justificando su pertinencia y enfatizando el empleo de sesiones grabadas en video junto con instrumentos de monitorización del resultado.

Palabras clave: Psicoterapia, PIR, Monitorización, Práctica, Deliberada, Entrenamiento.

\begin{abstract}
Despite the effectiveness of psychotherapy, there is evidence about its limitations and possibilities for improvement. Attending to the therapist it seems that clinical experience, traditional supervision and training in empirically supported treatments are not in themselves and separately a guarantee of greater efficacy.

Measures of routine outcome monitoring in psychotherapy have been designed and can increase its effectiveness. The use of these methods is relevant within a context of deliberate practice. This type of practice is characterized by setting goals and training in skills that exceed the current level of the practitioner. Within the psychotherapeutic field, deliberate practice can contribute to an improvement in the effectiveness of the therapist over time. This paper makes a review of these concepts and proposes a model of deliberate practice that can be applied to the Spanish PIR program context, justifying their relevance and emphasizing the use of video recording of sessions and instruments for outcome monitoring.
\end{abstract}

Keywords: Psychotherapy, PIR, Monitoring, Practice, Deliberate, Training.

Fecha de recepción: 4 de febrero de 2020. Fecha de aceptación: 3 de mayo de 2020.

Correspondencia sobre este artículo:

E-mail: pau.sanchez.rotger@hotmail.com

Dirección postal: Pau Sánchez Rotger. Calle Churruca, 2, 8 20, 28004 Madrid, España

(C) 2020 Revista de Psicoterapia 


\section{Contexto}

Según un meta-análisis reciente sobre la eficacia de la psicoterapia para pacientes con problemas de salud mental, el paciente que recibe psicoterapia mejora en término medio más que el $80 \%$ de aquellos que no la reciben (Wampold e Imel, 2015). No obstante, nos encontramos con un amplio margen de mejora en los resultados. Esto se justifica en dos hechos: el primero es que la eficacia no se ha incrementado desde el primer meta-análisis que evaluó los resultados de la psicoterapia (Smith y Glass, 1977), aún a pesar de la investigación, entrenamiento y aplicación de los tratamientos psicológicos basados en la evidencia (Asociación de Psicología Americana, 2012); y el segundo es que es común encontrar que hasta un 50\% de los pacientes no experimentan mejoría o incluso empeoran durante el tratamiento (Lambert, 2013). Además, uno de cada cuatro pacientes abandona prematuramente la psicoterapia antes de obtener resultados positivos (Swift y Greenberg, 2012).

Atendiendo a las variables relacionadas con el contexto de intervención, se ha encontrado que cuando se comparan tratamientos activos y estructurados que provienen de distintos modelos teóricos, estos son igualmente eficaces, lo que también se conoce como efecto del “pájaro dodo” (Wampold et al., 1997). Sin embargo, cuando se comparan unos terapeutas con otros las diferencias explican un mayor porcentaje de la variabilidad de los resultados de la psicoterapia (Baldwin e Imel, 2013; Saxon y Barham, 2012; Wampold y Brown, 2005).

Los programas de formación en psicoterapia parten generalmente del supuesto de que la experiencia clínica, la supervisión o la formación en tratamientos empíricamente validados conducen a una mejoría en los resultados del psicoterapeuta. Sin embargo, hay estudios que cuestionan una relación inequívoca entre la eficacia y la experiencia del terapeuta (Goldberg et al., 2016a; Owen, Wampold, Kopta, Rousmaniere y Miller, 2015). Respecto a la supervisión, a pesar de ser considerada como una variable central en la práctica clínica (Orlinsky, Botermans y Rønnestad, 2019), existe escasa literatura sobre su papel en la eficacia de la psicoterapia (Watkins, 2001; Rousmaniere, Babins-Wagner, Whipple y Barzins, 2014). Por último, un meta-análisis de 28 ensayos clínicos encontró que ni la adherencia a un tratamiento eficaz, entendida como ajuste a su protocolo de intervención, ni la competencia, entendida como la habilidad para implementarlo según los expertos en dicho tratamiento, se relacionaban de un modo significativo con una mayor eficacia de la psicoterapia (Webb, DeRubeis y Barber, 2010).

El programa de psicólogo interno residente (PIR) español tiene el objetivo, entre otros, de formar psicólogos clínicos capaces de llevar a cabo intervenciones psicoterapéuticas eficaces en un amplio rango de pacientes y dispositivos asistenciales (Olabarría y García, 2011). Para ello, el aspirante al programa PIR tiene que superar en primer lugar una exigente prueba tipo test de conocimientos relacionados con la psicología, especialmente en lo relativo a los tratamientos eficaces para trastornos psicológicos. Tras aprobar el examen de acceso, el PIR debe superar los cuatro años de residencia, a lo largo de los cuales rota por distintos dispositivos de 
la red de salud mental del sistema nacional de salud español. Uno de los aspectos centrales del programa es la práctica psicoterapéutica supervisada por psicólogos clínicos con mayor formación y experiencia, así como la formación continuada en aspectos relacionados con el ejercicio profesional. Así, el PIR dedica gran parte de las 37.5 horas semanales de trabajo a estos aspectos, habiendo acumulado un gran número de horas de práctica supervisada al final de su periodo formativo (para una revisión de la historia y el programa PIR, ver Prado-Abril, Sánchez-Reales, Gimeno Peon y Aldaz-Armendáriz, 2019).

Teniendo en cuenta la evidencia, parece que ninguna variable de las mencionadas conduce por sí misma y de forma inequívoca a una mejora en los resultados en psicoterapia. De este modo, el presente artículo propone una revisión de dos factores que han sido objeto de reciente investigación: la monitorización de los resultados y la práctica deliberada. Para ello, se defiende el contexto del sistema de residencia PIR como el adecuado para introducir estas variables, y se propone un modelo de práctica deliberada en base a la experiencia real en un hospital español.

\section{La monitorización de los resultados en psicoterapia}

En los últimos años se han creado sistemas que miden el progreso de la psicoterapia en tiempo real. Se trata de incluir en las sesiones de psicoterapia instrumentos de sencilla aplicación y que evalúan los resultados sesión a sesión, junto con otros aspectos relevantes del proceso terapéutico, como la alianza de trabajo. Esta práctica es conocida por sus siglas en inglés ROM (Routine Outcome Monitoring, o monitorización rutinaria del resultado) (Lambert, 2010). De entre los múltiple sistemas de monitorización del progreso del paciente que surgen en los últimos años, los instrumentos más utilizados en ensayos clínicos hasta la fecha son el Outcome Questionnaire System (OQ; Lambert et al., 1996) y el Partners for Change Outcome Management System (PCOMS; Miller, Duncan, Sorrel y Brown, 2005), ambos con propiedades psicométricas contrastadas (Bringhurst, Watson, Miller y Duncan, 2006; Miller, Duncan, Brown, Sparks y Claud, 2003; Reese, Toland y Kodet, 2012; Vermeersch et al., 2004).

-El OQ System contiene el instrumento OQ-45, una medida de auto-evaluación de 45 ítems que se emplea a sesión a sesión, evaluando el funcionamiento del paciente en las dimensiones de sintomatología (especialmente ansiedad y depresión), problemas interpersonales y desempeño social, junto con una subescala de calidad de vida.

-El PCOMS, por su parte, incluye dos escalas breves de cuatro ítems: a) el Outcome Rating Scale(ORS), cuyos ítems evalúan mediante un continuo las mismas dimensiones que el OQ; y b) el Session Rating Scale (SRS), cuyos ítems evalúan del mismo modo la alianza de trabajo, mediante las dimensiones de calidad en la relación con el terapeuta, satisfacción con las metas o temas abordados, con el enfoque o método empleado, y a nivel global, acorde con la conceptualización de alianza de trabajo de Bordin (Bordin, 1979). 
Un meta-análisis reciente de 24 estudios en los que se evaluaba la eficacia de estos instrumentos, encontró que en dos tercios de los estudios el grupo tratado con ROM mejoraba respecto al tratamiento habitual, con tamaños de efecto en el rango pequeño a moderado. El efecto fue mayor en aquellos pacientes a quienes se les pronosticaba un mal resultado, de forma que dentro de este grupo los tratados con ROM mejoraban hasta el doble respecto a los que no se les aplicaba el ROM (Lambert, Whipple y Kleinstäuber, 2018).

Una de las explicaciones sobre cómo el ROM puede incrementar la eficacia de la psicoterapia es que ayuda al profesional a ajustar sus impresiones sobre el progreso de sus pacientes mediante la observación rutinaria. De este modo, se ha encontrado que la capacidad de los terapeutas para predecir el progreso de sus pacientes es muy inferior a lo que estos creen (Hannan et al., 2005). Además, tienden a sobreestimar su propia eficacia en relación con sus pares. Walfish encontró que ninguno de 129 terapeutas que fueron encuestados se autoevaluó por debajo de la media, y hasta el $25 \%$ estimaban su propia pericia en el percentil 90 (Walfish, McAlister, O`Donnell y Lambert, 2012).

Otra explicación complementaria a la anterior hace referencia a la importancia de la alianza de trabajo. Se trata de uno de los factores que más investigación ha suscitado en la contribución a los resultados de la psicoterapia. De hecho, un metaanálisis de 190 estudios mostró que la alianza de trabajo explicaba hasta el 8\% de la variabilidad en los resultados. Este porcentaje era consistente entre estudios en los que se empleaban distintas fuentes, instrumentos y momentos de medida de la alianza, así como al seleccionar estudios cuyos autores la consideraban a priori un factor de importancia residual (Horvath, Del Re, Fluckiger y Symonds, 2011). Se ha encontrado además que es la contribución del terapeuta a la alianza de trabajo uno de los principales factores que explica la diferencia entre la eficacia de los terapeutas (Baldwin, Wampold e Imel, 2007). De este modo, es probable que aquellos terapeutas más eficaces lo sean en gran parte por su capacidad para establecer una sólida alianza de trabajo con un amplio rango de pacientes. En este caso, el ROM actuaría como medida de contraste ante la percepción del terapeuta, sujeta a los sesgos descritos, y la evolución real del progreso del paciente y la alianza de trabajo, permitiendo observar la diferencia entre ambas percepciones e introducir los cambios oportunos en el tratamiento de un modo colaborativo.

No obstante, es importante señalar que el ROM no contribuye en todos los casos a un aumento de la eficacia de la psicoterapia, como se evidencia en que hasta en un tercio de los estudios del meta-análisis mencionado no se encontraron diferencias favorables al ROM respecto al tratamiento habitual (Lambert et al., 2018). Otro meta-análisis en el que se incluyeron 18 estudios en los que se evaluaba la eficacia únicamente del sistema PCOMS, encontró un efecto nulo en aquellos estudios que tenían lugar en contextos psiquiátricos y con pacientes de mayor gravedad (Østergård, Randa y Hougaard, 2018). Una de las hipótesis de que la eficacia del ROM sea mayor en marcos comunitarios y con pacientes ambulatorios es que, 
en el contexto ambulatorio la colaboración y la alianza de trabajo son un factor central de la psicoterapia, mientras que en contextos psiquiátricos el tratamiento tiende a ser más estructurado y menos flexible a modificaciones. Esto también es aplicable a nivel del terapeuta: el ROM puede ser una herramienta útil en manos de un profesional abierto y responsivo a información que contradiga sus impresiones.

\section{Práctica deliberada en psicoterapia}

La práctica deliberada se define como "un entrenamiento individualizado en actividades especialmente diseñadas para mejorar aspectos concretos del rendimiento a través de la repetición y el refinamiento sucesivos" (Ericsson, 1996, pp. 278-279). Se diferencia de la práctica rutinaria de una disciplina en que su foco está en la adquisición de objetivos y habilidades por encima del nivel actual del individuo. Para ello, la práctica deliberada incluye el uso de medidas que objetiven que el progreso ha tenido lugar. De este modo, la práctica rutinaria facilita la automatización de las habilidades, aun siendo éstas mejorables. En contraste, la práctica deliberada se centra en flexibilizar y mejorar las habilidades actuales en base a un criterio de excelencia. Se ha investigado y aplicado la práctica deliberada en numerosas disciplinas como en música o medicina, y muchas de ellas cuentan actualmente con un método de práctica deliberada y evidencia sobre su papel en la mejora del rendimiento (para una revisión sobre el tema ver Ericcson, 2006).

La evidencia de la práctica deliberada en el campo de la psicoterapia es relativamente reciente pero con resultados favorables. Un estudio sobre la interacción entre las características de 69 terapeutas y sus diferencias en eficacia encontró que aquellos más eficaces dedicaban más tiempo a la mejora de aspectos específicos de su práctica psicoterapéutica, mediante por ejemplo el visionado de videos de sus sesiones (Chow et al., 2015). En otro estudio se obtuvo una interacción significativa entre la eficacia de una muestra de 70 terapeutas y sus autoevaluaciones en autocuestionamiento profesional ("profesional self-doubt"). Concretamente, aquellos terapeutas que mostraban altos niveles de auto-cuestionamiento profesional junto con altos niveles de auto-afirmación eran consistentemente más eficaces (NissenLie et al., 2015). Así, estos datos sugieren que para que el psicoterapeuta pueda reflexionar sobre sus aspectos a mejorar de una forma continuada y constructiva, es importante reforzar los aspectos positivos del self, y para ello cobra especial relevancia que el aprendizaje tenga lugar en un contexto seguro.

A pesar de la evidencia de la contribución de la práctica deliberada a la mejora del rendimiento en otras disciplinas, la psicoterapia ha tardado en adaptar y elaborar un método de este tipo de práctica en este campo. Esto se debe probablemente a varias razones. En primer lugar, a diferencia de otras disciplinas, la psicoterapia tiene lugar generalmente en la intimidad del despacho, de forma que el trabajo de los psicoterapeutas no suele ser objeto de observación y evaluación. Así, el método tradicional de supervisión consiste en que el supervisando expone el caso al supervisor atendiendo al contenido de su memoria y, en el mejor de los casos, a 
anotaciones en la historia clínica. Por tanto, el material de trabajo ya se encuentra sujeto a omisiones, distorsiones y adiciones respecto a lo ocurrido en la sesión. En segundo lugar, en psicoterapia no hay un estándar de excelencia fácilmente reconocible por parte de la comunidad, como puede haberlo en música, deportes o cirugía. De hecho, como se ha visto anteriormente, no hay evidencia de que los que son considerados expertos en un determinado modelo obtengan consistentemente mejor resultado con sus pacientes (Webb et al., 2010). Por último, el objeto de trabajo psicoterapéutico es la propia díada paciente (o pareja, familia) y terapeuta implicada en el proceso. Es por tanto un trabajo en el que intervienen muchas variables pertenecientes a cada una de las partes y a la interacción. De este modo, resulta más complejo establecer una rutina de práctica deliberada en solitario, en comparación por ejemplo con una simulación quirúrgica o con el ensayo de un instrumento musical.

A pesar de las limitaciones descritas, Rousmaniere introdujo un modelo de práctica deliberada aplicable a la psicoterapia, que se estructura en estos cinco aspectos (Rousmaniere, 2016):

1) Observar el trabajo psicoterapéutico y establecer una línea base de la propia eficacia.

2) Conseguir supervisión por parte de un experto.

3) Ponerse metas cercanas al propio nivel de habilidad.

4) Practicar repetidamente.

5) Evaluar de forma constante el progreso.

Se ha realizado un estudio prospectivo sobre el cambio en la eficacia del terapeuta a lo largo del tiempo (Goldberg et al., 2016b), similar al mencionado anteriormente (Goldberg et al., 2016a). Sin embargo, en este estudio se aplicaron sistemáticamente las recomendaciones descritas en el modelo propuesto por Rousmaniere y otros autores (Tracey, Wampold, Lichtenberg y Goodyear 2014). El análisis de 153 psicoterapeutas que atendieron un total de 5128 pacientes durante siete años mostró, contrariamente al estudio anterior, una mejoría en la eficacia media a nivel de la organización de salud mental y de los terapeutas, con un incremento en el tamaño del efecto de $d=0.034$ cada año (Goldberg et al., 2016b).

\section{Práctica deliberada en el programa PIR español}

Como se ha expuesto, la superación del programa de residencia PIR conlleva haber adquirido unos conocimientos teóricos y experiencia práctica supervisada que son de gran valor para el psicólogo clínico, y garantiza unos mínimos de atención de calidad de cara al trabajo psicoterapéutico. Además, la experiencia de residente durante cuatro años aporta conocimientos teóricos y prácticos de gran utilidad en otros aspectos no directamente relacionados con la psicoterapia, como por ejemplo en el ámbito de la toma de decisiones clínicas en el contexto del sistema nacional de salud. No obstante, se ha expuesto que ni el entrenamiento en tratamientos empíricamente validados, ni la experiencia ni la supervisión constituyen por sí 
mismos condiciones suficientes para un aumento de la eficacia en la psicoterapia. Paralelamente, se ha resumido la evidencia disponible en lo que respecta al ROM y a la práctica deliberada en psicoterapia, y su contribución al aumento de su eficacia. La práctica deliberada en el programa PIR español ha sido objeto reciente de reflexiones (Prado-Abril, Sánchez-Reales e Inchausti, 2017; Prado-Abril, Gimeno Peón, Sánchez-Reales, 2019; Revenga Montejano y Martín Garcia, 2019).

De hecho, los autores el presente artículo defienden que el contexto de trabajo supervisado a lo largo de cuatro años del programa PIR es el indicado para plantear propuestas de práctica deliberada por varias razones: en primer lugar, el hecho de que el residente rote por distintos dispositivos y con pacientes con distinta problemática obliga a una continua revisión y ajuste de las propias habilidades terapéuticas en contextos diversos; en segundo lugar, la supervisión por distintos terapeutas facilita que el residente reciba un feedback variado acerca de su propio desempeño y distintos modelos de trabajo, lo cual contribuye a estimular su autoreflexión; y en tercer lugar, los espacios de docencia reglada proporcionan las condiciones para estimular esta práctica dada su integración en la jornada laboral y práctica psicoterapéutica.

A continuación se propone una adaptación del modelo de práctica deliberada de Rousmaniere (Rousmaniere, 2016) aplicable al contexto del programa de residencia PIR, atendiendo a cada uno de sus aspectos:

1) Observar el trabajo psicoterapéutico y establecer una línea base de la propia eficacia. Para poder mejorar es necesario conocer de dónde se parte. El residente no suele tener información sobre su nivel de eficacia al iniciar, por ejemplo, su rotación en un Centro de Salud Mental. Para ello, la incorporación del ROM a la práctica diaria puede ayudarle a contrastar el grado en que sus casos progresan según lo esperado. Como se ha expuesto, sistemas como el PCOMS proporcionan medidas breves y sencillas de aplicar sobre el resultado de la psicoterapia y la alianza de trabajo.

2) Conseguir supervisión por parte de un experto. En relación con este punto y el anterior, el residente tampoco suele tener información sobre su propio desempeño dentro de la sesión, salvo solo a través de su memoria. Se han comentado las limitaciones de la supervisión tradicional en tanto que se parte de lo percibido y recordado por el propio supervisando. La grabación de las sesiones clínicas y la supervisión de las mismas puede ofrecer información al residente y a su supervisor sobre su actuación desde otra perspectiva. Este método de supervisión en vídeo debería incorporarse junto a la supervisión tradicional y la formulación de caso en los espacios de supervisión y docencia del período de formación PIR. Una alternativa al vídeo es la grabación en audio de la sesión, que puede resultar más sencilla y menos ansiógena tanto para el terapeuta como para el paciente, a la vez que recoge gran parte de la información conversacional relevante 
de cara a la supervisión.

En este punto también es necesario reflexionar sobre quién constituye un experto en psicoterapia. Dada la insuficiencia de datos sobre el desempeño real de los psicoterapeutas, una posibilidad es seleccionar a un supervisor en función de la consideración del residente sobre su habilidad psicoterapéutica, junto con los criterios de accesibilidad y disponibilidad inherentes al programa de residencia. Es común en los residentes la impresión de que determinados profesionales cuentan con un repertorio de conocimientos y habilidades muy superiores a las propias, y quizás estos sean elegibles en la labor de supervisor.

Una variable relevante en este sentido es el estilo personal del terapeuta, definido como "un conjunto de condiciones singulares que llevan a un terapeuta a operar de una manera particular en su trabajo” (Fernández-Álvarez y García, 1998). Se trata de un constructo multidimensional que incluye el modo de establecer el encuadre, la expresividad emocional, el grado de compromiso con su trabajo, el modo de atender o seleccionar la información en la sesión y las acciones o intervenciones específicas. Se evalúa mediante el cuestionario de estilo personal del terapeuta (EPT-C) (Fernández-Álvarez, García, Lo Bianco y Corbella, 2003), recientemente validado en población española (Prado-Abril et al., 2020). Se ha comparado el estilo personal del terapeuta en función de la escuela psicoterapéutica (Quiñones y Ugarte, 2019) o experiencia atendiendo a un tipo de problemática (Casari e Ison, 2019). Una hipótesis a contrastar sería que un supervisor cuyo estilo terapéutico fuese similar al del residente favoreciese su aprendizaje, a través de un marco compartido de actitudes y conductas respecto a la supervisión y el proceso terapéutico. En tal caso podría considerarse este criterio en la selección del supervisor.

3) Ponerse metas cercanas pero superiores al propio nivel de habilidad. En el espacio de supervisión en vídeo, el supervisor puede seleccionar una o varias escenas clínicas relevantes, ya sea por su importancia en el proceso psicoterapéutico o porque ilustran una habilidad concreta que el residente puede mejorar. Tras exponer esta habilidad y la justificación de su pertinencia, puede tener lugar un role-playing en el que el supervisor muestre la habilidad interpretando el papel de terapeuta, para que después el residente practique esta habilidad y reciba las correcciones pertinentes.

Es importante seleccionar habilidades que se encuentren dentro de la zona de desarrollo próximo del residente (Vygotsky, 1978), y en un contexto cálido, seguro y que facilite el aprendizaje.

4) Practicar repetidamente. Una vez realizada la supervisión según el formato mencionado, el residente tiene oportunidades para practicar las habilidades aprendidas, tanto en sesiones posteriores con sus pacientes, como en roleplaying o incluso en solitario, ayudándose del vídeo como estímulo con el que interactuar. Como guía de habilidades a practicar, en una muestra de 25 terapeutas se observó que aquellos que tenían altas puntuaciones en las llamadas habilidades de facilitación interpersonal obtenían mejores 
resultados con sus pacientes (Anderson, Ogles, Patterson, Lambert y Vermeersch, 2009). Estas habilidades son la fluidez verbal, la expresión de un amplio rango de emociones, la capacidad de persuasión y esperanza, la calidez y empatía, la capacidad para formar una sólida alianza de trabajo y la orientación hacia el problema. La práctica de estas habilidades era especialmente relevante en situaciones clínicas complejas, como por ejemplo ante un paciente enfadado, retraído o autopunitivo (Anderson et al., 2009).

Por otra parte, existen clasificaciones sobre habilidades terapéuticas relacionadas con variables relevantes en la psicoterapia: con la estructura del tratamiento, por ejemplo, cómo cerrar una sesión o cómo elicitar feedback del paciente; con el fomento de expectativas de mejora, por ejemplo, cómo expresar confianza en el tratamiento o proporcionar una explicación al problema del paciente en términos adaptados; con la construcción de una alianza de trabajo, por ejemplo, cómo movilizar la preparación al cambio, promover un vínculo cálido o abordar una ruptura en la alianza; con lo relacionado con el self del terapeuta, por ejemplo, cómo manejar la contratransferencia, regular la propia ansiedad o emplear auto-revelaciones; y con las variables del propio paciente, por ejemplo, cómo reforzar sus fortalezas y adecuar el tratamiento a sus valores y creencias (Chow et al., 2015).

La práctica de éstas y otras habilidades de forma deliberada implica un proceso opuesto a la práctica rutinaria, ya que mientras ésta conduce a la progresiva automatización de la conducta del terapeuta, aquella está dirigida a la toma de conciencia y flexibilización del repertorio conductual de habilidades, para poder adaptarse a un amplio rango de situaciones clínicas y pacientes.

5) Evaluar de forma constante el progreso. Para lograr un incremento progresivo en la eficacia de la psicoterapia, es importante realizar una evaluación constante del propio desempeño. Sólo así se puede comprobar si las habilidades practicadas y los temas abordados en consulta contribuyen a una mejoría clínica, sin menoscabo de la importancia de los factores extraterapéuticos (Norcross y Goldfried, 2019). Para ello es fundamental incorporar el ROM a la práctica diaria. De hecho, se ha observado un incremento de la eficacia de una muestra de 20 terapeutas a lo largo de los años tras la introducción del ROM (Brattland et al., 2018).

Además, los instrumentos mencionados pueden complementarse con otros métodos que aporten una visión más amplia y rica sobre la perspectiva del paciente. Por ejemplo, se puede visionar el video de una sesión con el propio paciente y pedirle que señale los momentos más relevantes para él y sus motivos. Esto permite el acceso a su perspectiva momento a momento, una fuente de información de indudable valor en el proceso (Kagan, 1980; Bernard, 1989). 


\section{Una experiencia piloto}

Durante el curso 2018-19 se implementó dentro del programa de docencia para residentes de psicología clínica y psiquiatría del H.U. La Paz de Madrid un taller dirigido a la mejora de las habilidades como psicoterapeutas de los residentes, siguiendo el modelo de práctica deliberada descrito. Con frecuencia bimestral, se realizaron seis sesiones a lo largo del curso en formato grupal, incluyendo a todos los residentes disponibles. La duración fue de una hora, y la participación como supervisando voluntaria. Durante este primer año todos los voluntarios se encontraban en uno de los dos últimos años de residencia y trabajaron sobre entrevistas de psicoterapia de pacientes en seguimiento en ambulatorio. La estructura del taller se describe a continuación:

1. Trabajo pre-sesión: el supervisando envía la grabación de la entrevista al supervisor, indicándole los aspectos de la misma con los que no se siente satisfecho. El supervisor, por su parte, revisa la grabación, identifica una habilidad a mejorar y selecciona uno o varios fragmentos breves (no más de 2 minutos) en los que la ejecución de la misma era mejorable. Supervisor y supervisando acuerdan a partir de ese momento la habilidad a trabajar en la sesión. Pese a que no se incluía en la propuesta inicial, la revisión de las entrevistas llevó a que el supervisor decidiera seleccionar también algún fragmento breve que ilustrase habilidades terapéuticas bien ejecutadas en la misma sesión con intención de potenciar el componente motivador y disminuir el temor del profesional a la exposición grupal de sus dificultades en las sesiones.

2. Trabajo en sesión: la sesión comienza con el residente haciendo una muy breve contextualización del caso para el grupo, así como un resumen general de la entrevista y las dificultades que se le presentan. El supervisor introduce la habilidad a trabajar y la explica de forma resumida, ayudándose en ocasiones con elementos audiovisuales. Se realiza la visualización de los fragmentos de video y se señalan los aspectos de mejora. En este punto se hace una breve pausa por si hay necesidad de comentarios o aclaraciones y se pasa a la práctica de la habilidad. En formato de role playing, el supervisor ejerce el papel de terapeuta, modelando la ejecución de la habilidad, y el residente desempeña el rol de paciente. Si se dispone de tiempo, se invierten los papeles o se propone una práctica por parejas entre todos los miembros del grupo. La sesión concluye con un intercambio de impresiones respecto a lo trabajado y con indicaciones de práctica de la habilidad en su desempeño habitual.

En términos generales, la dificultad más habitualmente observada en los terapeutas en formación es una excesiva presión acerca de la propia ejecución y una elevada autofocalización por parte de los residentes, que les dificulta estar receptivos, presentes y centrados en las personas a las que atienden, factor en el que pueden pesar tanto variables estables como 
la escasa experiencia o el competitivo entorno hospitalario, y variables situacionales como el contexto de grabación para la supervisión. Como habilidades más destacables se aprecian su capacidad para favorecer el vínculo, para el análisis de conflictos y una elevada disposición personal. Las habilidades trabajadas en las distintas sesiones de 2018-19 fueron:

- Trabajo con momentos de ambivalencia: tolerancia, exploración, formulación (Oliveira, Gonçalves, Braga y Ribeiro, 2016)

- Rupturas y reparaciones vinculares: detección y alternativas técnicas (Safran y Krauss, 2014)

- Trabajo sobre excepciones en narrativas dominantes (De Shazer, 1988)

- Mantenimiento de la distancia terapéutica y funcionamiento reflexivo trabajando con estilos de apego ambivalentes-resistentes (Baim, Morison y Hathaway, 2011)

- Intervención ante indicadores de evitación experiencial(Muller, 2010)

- Reconstrucción y despliegue de episodios conflictivos (Dimaggio, Montano, Popolo y Salvatore, 2015)

A modo de ejemplo detallaremos el trabajo con esta última habilidad: Durante la entrevista hay varios momentos en que la paciente hace un relato fragmentado, parcial, de episodios vitales recientes y se muestra poco receptiva a la exploración. La fragmentación narrativa parece esconder vivencias y afectos poco integrados en su conciencia, y probablemente relevantes en cuanto a sus conflictos principales. El terapeuta se maneja principalmente en un plano semántico, tratando de dotar de un sentido general a la experiencia, y prescriptivo, buscando soluciones a problemas poco definidos, pero se siente desarmado. Se propone y modela un trabajo alternativo focalizado en el despliegue detallado de los episodios y la autoobservación por parte de la paciente, con el objetivo de que pueda contactar con estas vivencias y la información relevante para su autoorganización que contienen, tal y como proponen técnicas como el Despliegue evocador sistemático (Rice y Saperia 1984), la Moviola (Guidano, 1995) o el despliegue de episodios en el trabajo con trastornos de personalidad (Dimaggio et al., 2015).

3. Trabajo post-sesión: los residentes practican en sus contextos profesionales, en un ambiente natural y revisan su experiencia en los entornos de supervisión de los que disponen.

Tras este primer bloque de trabajo y coincidiendo con la incorporación de los nuevos residentes al taller, se realiza un grupo de discusión sobre el funcionamiento del mismo. Los recién incorporados viven la posibilidad de exponerse con un elevado componente de temor, dada su escasa experiencia, mientras que los que han pasado por el proceso y han trabajado en sesiones anteriores transmiten una sensación de aprendizaje y tratan de transmitir una cultura de trabajo abierto y seguridad. Como posible elemento de mejora para próximas ediciones se plantean 
las siguientes opciones:

- Aumentar el tiempo de duración en media hora para garantizar la práctica de todos los asistentes en los role playings.

- Dividir el grupo en dos, de modo que aumente la contención, disminuya el grado de exposición y se permita una mayor implicación de todos los participantes

- Valorar la posibilidad de hacer un trabajo diferenciado: habilidades generales de entrevista y facilitación interpersonal en los dos primeros años de residencia, y habilidades específicas con mayor contenido técnico en el tercer y cuarto año.

\section{Conclusiones}

A pesar de la eficacia establecida de la psicoterapia para pacientes con problemas de salud mental, existe evidencia sobre su potencial de mejora. Además, parece que la diferencia entre terapeutas explica una mayor variabilidad en los resultados que la diferencia entre tratamientos. Al considerar las características del terapeuta, las variables como experiencia clínica, supervisión o formación en tratamientos basados en la evidencia no son condiciones suficientes para una mayor eficacia. En este sentido, la introducción de instrumentos de monitorización del progreso terapéutico o ROM puede contribuir a mejorar los resultados del tratamiento, al proporcionar información sesión a sesión que permita introducir modificaciones y adaptarse a las necesidades del paciente. El uso del ROM debe tener lugar en un contexto de práctica deliberada, un tipo de práctica específicamente centrada en el establecimiento de objetivos y práctica de habilidades que se encuentren en un nivel superior al actual. De este modo, el programa PIR constituye una oportunidad única para la práctica deliberada, al tratarse de un contexto seguro con numerosos espacios para la reflexión y supervisión. Además, en numerosas ocasiones la residencia coincide con el inicio de la práctica profesional en la psicología clínica, cobrando de este modo especial importancia realizar una práctica reflexionada y centrada en los aspectos a mejorar. La grabación de sesiones clínicas, la supervisión y práctica de habilidades terapéuticas, junto con el uso del ROM son aspectos que deberían incorporarse a los programas de residencia PIR español, con el objetivo de contribuir a una mayor eficacia en las habilidades psicoterapéuticas del psicólogo clínico durante y después del período de residencia.

\section{Referencias Bibliográficas}

American Psychological Association (2012, agosto 9). Resolution on the recognition of psychotherapy effectiveness. Recuperado de: https://www.apa.org/about/policy/resolution-psychotherapy

Anderson, T., Ogles, B. M., Patterson, C. L., Lambert M. J. y Vermeersch, D. A. (2009). Therapist effects: Facilitative interpersonal skills as a predictor of therapist success. Journal of Clinical Psychology, 65(7), 755-768. https://doi.org/10.1002/jclp.20583 
Baim, C., Morrison, T. y Hathaway, J. (2011). Attachment-based Practice with Adults: Understanding Strategies and Promoting Positive Change: Anew practice model and interactive resource for assessment, intervention and supervision. Brighton, Reino Unido: Pavilion.

Baldwin, S. A. e Imel, Z. E. (2013). Therapist effects: Findings and methods. En M. J. Lambert (Ed.), Bergin and Garfield's handbook of psychotherapy and behavior change (6a ed., pp. 258-297). Nueva York, NY: Wiley.

Baldwin, S. A., Wampold, B. E. e Imel, Z. E. (2013). Untangling the alliance-outcome correlation: exploring the relative importance of therapist and patient variability in the alliance. Journal of Consulting and Clinical Psychology, 75(6), 842-852. https://doi.org/10.1037/0022-006X.75.6.842

Bernard, J. M. (1989). Training supervisors to examine relationship issues using IPR. The Clinical Supervisor, 7(1), 103-112. https://doi.org/10.1300/J001v07n01_08

Bordin, E. S. (1979). The generalizability of the psychoanalytic concept of the working Alliance. Psychotherapy: Theory, Research, \& Practice, 16(3), 252-260. https://doi.org/10.1037/h0085885

Brattland, H., Koksvik, J. M, Burkeland O., Grave, R. W., Klöckner, C., Linaker, O. M. ... Iversen, V. C. (2018). The effects of routine outcome monitoring (ROM) on therapy outcomes in the course of an implementation process: A randomized clinical trial. J Couns Psychol, 65(5), 641-652. https://doi.org/10.1037/cou0000286

Bringhurst, D. L., Watson, C. W., Miller, S. D. y Duncan, B. L. (2006). The reliability and validity of the Outcome Rating Scale: A replication study of a brief clinical measure. Journal of Brief Therapy, 5(1), 23-30. Recuperado de: https://www.scottdmiller.com/wp-content/uploads/JBTORSReplication.pdf

Casari, L. e Ison, M. S. (2019). Estilo Personal del Terapeuta: comparación entre profesionales que trabajan en instituciones de adicciones y profesionales del área clínica general [Therapist Personal Styles: AComparison Between Professionals Working in Drug Addiction Institutions and Professionals Working in the General Clinical Area]. Revista Costarricense de Psicología, 38(1), 57-73. https://doi.org/10.22544/rcps.v38i01.04

Chow, D. L., Miller, S. D., Seidel, J. A., Kane, R. T., Thornton, J. A. y Andrews, W. P. (2015). The role of deliberate practice in the development of highly effective psychotherapists. Psychotherapy, 52(3), 337-345. https://doi.org/10.1037/pst0000015

De Shazer, S. (1988). Clues: Investigating solutions in brief therapy. Londres, Reino Unido: WW Norton \& Co.

Ericsson, K. A. (2006). The Influence of experience and deliberate practice on the development of superior expert performance. En K. A. Ericsson, N. Charness, P. J. Feltovich y R. R. Hoffman (Eds.), The Cambridge handbook of expertise and expert performance (pp. 683-704). Nueva York, NY: Cambridge University Press.

Dimaggio, G., Montano, A., Popolo, R. y Salvatore, G. (2015). Metacognitive interpersonal therapy for personality disorders: A treatment manual. Londres, Reino Unido: Routledge.

Fernández-Álvarez, H. y Garcia, F. (1998). El Estilo Personal del Terapeuta: Inventario para su evaluación. En S. Gril, A. Ibáñez, I. Mosca y P. L. R. Sousa (Eds.), Investigación en Psicoterapia (pp.76-84). Pelotas, Brasil: Educat.

Fernández-Álvarez, H., García, F., Lo Bianco, J. y Corbella, S. (2003). Assesment Questionnaire on The Personal Style of the Therapist PST-Q. Clinical Psychology and Psychotherapy, 10(2), 116-125. https://doi. org/10.1002/cpp.358

Goldberg, S. B., Rousmaniere, T., Miller, S., Whipple, J, Nielsen, S. L., Hoyt, W. T. y Wampold, B. E. (2016a). Do psychotherapists improve with time and experience? A longitudinal analysis of outcomes in a clinical setting. Journal of Counseling Psychology, 63(1), 1-11. https://doi.org/10.1037/cou0000131

Goldberg S. B., Babins-Wagner R., Rousmaniere T., Berzins S., Hoyt W. T., Whipple J. L. y Wampold B. E. (2016b). Creating a climate for psychotherapist improvement: A case study of an agency focused on outcomes and deliberate practice. Psychotherapy, 53(3), 367-375. https://doi.org/10.1037/pst0000060

Guidano, V. (1995). A constructivist outline of human knowing processes. En Mahoney, M. J. (Ed.), Cognitive and constructive psychotherapies: Theory, research, and practice (pp. 89-102). Nueva York, NY: Springer.

Hannan, C., Lambert, M., Harmon, C., Nielsen, S. L., Smart, D. W., Shimokawa, K. y Sutton, S. W. (2005). A lab test and algorithms for identifying clients at risk for treatment failure. Journal of Clinical Psychology, 61(2), 155-163. https://doi.org/10.1002/jclp.20108

Horvath, A. O., Del Re, A. C., Fluckiger, C. y Symonds, D. (2011). Alliance in Individual Psychotherapy. Psychotherapy, 48(1), 9-16. https://doi.org/10.1037/a0022186

Kagan, N. (1980). Influencing human interaction-Eighteen years with IPR. En A.K. Hess (Ed.), Psychotherapy supervision: Theory, research, and practice (pp. 262-283). Nueva York, NY: Wiley

Lambert, M. J. (2010). Prevention of treatment failure: The use of measuring, monitoring, and feedback in clinical practice. Washington, DC: American Psychological Association.

Lambert, M. J. (2013). The efficacy and effectiveness of psychotherapy. En M. J. Lambert (Ed.), Bergin and Garfield's handbook of psychotherapy and behaviour change (6 $\left.{ }^{\mathrm{a}} \mathrm{ed} ., \mathrm{pp} .169-218\right)$. Hoboken, NJ: JohnWiley. 
Lambert, M. J., Hansen, N. B., Umphress, V. J., Lunnen, K., Okiishi, J., Burlingame, G. y Reisinger, C. W. (1996). Administration and scoring manual for the outcome questionnaire (OQ 45.2). Wilmington, DE: American Professional Credentialing Services

Lambert, M. J., Whipple, J. L. y Kleinstäuber, M. (2018). Collecting and delivering progress feedback: A metaanalysis of routine outcome monitoring. PsychoTherapy55(4), 520-537. https://doi.org/10.1037/pst0000167

Miller, S. D., Duncan, B. L., Brown, J., Sparks, J. y Claud, D. (2003). The outcome rating scale: A preliminary study of the reliability, validity, and feasibility of a brief visual analogue measure. Journal of Brief Therapy, 2(2), 91-100. Recuperado de: https://scottdmiller.com/wp-content/uploads/documents/OutcomeRatingScaleJBTv2n2.pdf

Miller, S. D., Duncan, B. L., Sorrell, R. y Brown, G. S. (2005) The partners for change outcome management system. J Clinical Psychology, 61(2), 199-208. https://doi.org/10.1002/jclp.20111

Muller, R. T. (2010). Trauma and the avoidant client: Attachment-based strategies for healing. Nueva York, NY: WW Norton \& Company.

Nissen-Lie, H. A., Rønnestad, M. H., Høglend, P. A., Havik, O. E., Solbakken, O. A., Stiles, T. y Monsen, J. T. (2015). Love Yourself as a Person, Doubt Yourself as a Therapist? Clinical Psychology, Psychotherapy, 24(1), 48-60. https://doi.org/10.1002/cpp.1977

Norcross, J. C. y Goldfried, M. R, (2019). Handbook of Psychotherapy Integration (3 $3^{\mathrm{a} e d .) . ~ N u e v a ~ Y o r k, ~ N Y: ~}$ Oxford University press.

Olabarría, B. y García, M. A. (2011). Acerca del proceso de construcción de la Psicología Clínica en España como Especialidad Sanitaria [On the construction of clinical psychology in Spain as a health specialty]. Revista de Psicopatologíay Psicología Clínica, 16(3), 223-245. https://doi.org/10.5944/rppc.vol.16.num.3.2011.10363

Oliveira, J. T., Gonçalves, M. M., Braga, C. y Ribeiro, A. P. (2016). Cómo lidiar con la ambivalencia en psicoterapia: un modelo conceptual para la formulación de caso [How to deal with ambivalence in psychotherapy: a conceptual model for case formulation]. Revista de psicoterapia, 27(104), 83-100. https://doi. org/10.33898/rdp.v27i104.122

Orlinsky, D. E., Botermans, J. F. y Rønnestad, M. H. (2001). Towards an Empirically Grounded Model of Psychotherapy Training: Four Thousand Therapists Rate Influences on Their Development. Australian Psychologist, 36(1), 139-148. https://doi.org/10.1080/00050060108259646

Østergård, O. K, Randa, H. y Hougaard, E. (2018). The effect of using the partners for change outcome management system as feedback tool in psychotherapy: A systematic review and meta-analysis. Psychotherapy Research, 30(2), 195-212. https://doi.org/10.1080/10503307.2018.1517949

Owen, J., Wampold, B. E., Kopta, M., Rousmaniere, T. y Miller, S. D. (2015). As good as it gets? Therapy outcomes of trainees over time. Journal of Counseling Psychology, 63(1), 12-19. https://doi.org/10.1037/cou0000112

Prado-Abril, J., Fernández-Álvarez, J., Sánchez-Reales, S., Youn, S. J., Inchausti, F. y Molinari, G. (2020). La persona del terapeuta: Validación española del Cuestionario de Evaluación del Estilo Personal del Terapeuta (EPT-C). Revista de Psicopatologíay Psicología Clínica, 24(3), 131-149. https://doi.org/10.5944/rppc.24367

Prado-Abril, J., Gimeno-Peón, A., Inchausti, F. y Sánchez-Reales, S. (2019). Expertise, therapist effects and deliberate practice: The cycle of excellence. Papeles del Psicólogo, 40(2), 89-100. https://doi.org/10.23923/ pap.psicol2019.2888

Prado-Abril, J., Sánchez-Reales, S., Gimeno Peón, A. y Aldaz-Armendáriz, J. A. (2019). Clinical Psychology in Spain: History, Regulation and Future Challenges, 1(4). 1-12. https://doi.org/10.32872/cpe.v1i4.38158

Prado-Abril, J., Sánchez-Reales, S. e Inchausti, F. (2017). En busca de nuestra mejor versión: pericia y excelencia en Psicología Clínica. Ansiedad y Estrés, 23(2-3), 110-117. https://doi.org/10.1016/j.anyes.2017.06.001

Quiñones, Á. y Ugarte, C. (2019). Investigación del estilo personal del terapeuta post-racionalista: Una revisión [Research of the personal style of the post-rationalist therapist: a review]. Revista De Psicoterapia, 30(112), 7-15. https://doi.org/10.33898/rdp.v30i112.281

Reese, R. J., Toland, M. D. y Kodet, J. (2012, Agosto). Validity of a psychotherapy outcome measure: The Outcome Rating Scale. Sesión de Póster presentado en The annual meeting of the American Psychological Association, Orlando, FL.

Revenga Montejano, S. y Martín García, A. (2019). Reflexiones sobre la formación en Psicología Clínica: el camino hacia la Pericia. Revista clínica contemporánea, 10(3), 1-15. https://doi.org/10.5093/cc2019a19

Rice, L. N. y Saperia, E. (1984). A task analysis of the resolutions of problematic reactions. En L. N. Rice y L. S. Greenberg (Eds.), Patterns of change: Intensive analysis of psychotherapy process (pp. 29-66). Nueva York, NY: Guilford press.

Rousmaniere, T. (2016). Deliberate Practice for Psychotherapists: A guide to improving clinical effectiveness. Nueva York, NY: Routledge. 
Rousmaniere, T. G., Swift, J. K., Babins-Wagner, R., Whipple, J. L. y Barzins, S. (2014). Supervisor variance in psychotherapy outcome in routine practice. Psychotherapy Research, 26(2), 196-205. https://oi.org/10. 1080/10503307.2014.963730

Safran, J. D. y Kraus, J. (2014). Alliance ruptures, impasses, and enactments: A relational perspective. Psychotherapy, 51(3), 381-387. https://doi.org/10.1037/a0036815

Saxon, D. y Barkham, M. (2012). Patterns of therapist variability: Therapist effects and the contribution of patient severity and risk. Journal of Consulting and Clinical Psychology, 80(4), 535-546. https://doi.org/10.1037/ a0028898

Smith, M. L. y Glass, G. V. (1977). Meta-analysis of psychotherapy outcome studies. American Psychologist, 32(9), 752-760. https://doi.org/10.1037/0003-066X.32.9.752

Swift, J. K. y Greenberg, R. P. (2012). Premature discontinuation in adult psychotherapy: A meta-analysis. Journal of Consulting and Clinical Psychology, 80(4), 547-559. https://doi.org/10.1037/a0028226

Tracey, T. J. G., Wampold, B. E., Lichtenberg, J. W. y Goodyear, R. K. (2014). Expertise in psychotherapy: An elusive goal? American Psychologist, 69(3), 218-229. https://doi.org/10.1037/a0035099

Vermeersch, D. A., Whipple, J. L., Lambert, M. J., Hawkins, E. J., Burchfield, C. M. y Okiishi, J. C. (2004). Outcome Questionnaire: Is it sensitive to changes in counseling center clients? Journal of Counseling Psychology, 51(1), 38-49. https://doi.org/10.1037/0022-0167.51.1.38

Vygotsky, L. S. (1978). Mind in Society: the Development of Higher Psychological Processes. Cambridge, MA: Harvard University Pres.

Walfish, S., McAlister, B., O’Donnell, P. y Lambert, M. (2012). An Investigation of Self-Assessment Bias in Mental Health Providers. Psychological reports, 110(2), 639-644. https://doi.org/10.2466/02.07.17. PR0.110.2.639-644

Wampold, B. E. y Brown, G. S. (2005). Estimating varibility in outcomes attributable to therapists: a naturalistic study of outcomes in managed care. Journal of Clinical Psychology and Clinical Psychology, 73(5), 914923. https://doi.org/10.1037/0022-006X.73.5.914

Wampold, B. E. e Imel, Z. E. (2015). The Great Psychotherapy Debate: The evidence fot What makes Psychotherapy Work. Nueva York, NY: Routledge.

Wampold, B. E., Mondin, G. W., Moody, M., Stich, F., Benson, K. y Ahn, H.-n. (1997). A meta-analysis of outcome studies comparing bona fide psychotherapies: Empirically, "all must have prizes". Psychological Bulletin, 122(3), 203-215. https://doi.org/10.1037/0033-2909.122.3.203

Watkins, C. E. (2001). Does psychotherapy supervision contribute to patient outcomes? Considering 30 years of research. The clinical supervisor, 30(2), 235-256. https://doi.org/10.1080/07325223.2011.619417

Webb, C. A., DeRubeis, R. J. y Barber, J. P. (2010). Therapist adherence/competence and tratment outcome: A meta-analytic review. Journal of Consulting and Clinical Psychology, 78(2), 200-211. https://doi. org/10.1037/a0018912 